\title{
Trends of surgical treatment for spinal degenerative disease in China: a cohort of 37,897 inpatients from 2003 to 2016
}

This article was published in the following Dove Medical Press journal:

Clinical Interventions in Aging

Yan $\mathrm{Li}^{1, *}$

Si Zheng 2 ,*

Yunxia $\mathrm{Wu}^{1, *}$

Xiaoguang Liu'

Gengding Dang'

Yu Sun'

Zhongqiang Chen'

Jiayang Wang ${ }^{2}$

Jiao $\mathrm{Li}^{2}$

Zhongjun Liu'

'Department of Orthopaedics, Peking University Third Hospital, Beijing, China; 'Institute of Medical Information, Chinese Academy of

Medical Sciences, Peking Union

Medical College, Beijing, China

*These authors contributed equally to this work
Correspondence: Zhongjun Liu Department of Orthopaedics, Peking University Third Hospital, No 49 Hua Yuan Bei Road, Beijing 100191, China

Tel/fax +86 I0 82267338

Email prof_zhongjunliu@126.com
Purpose: Given the aging Chinese population and the inevitable degenerative process of the spine, more elderly patients with spinal degenerative disease (SDD) are surgical candidates, placing a significant burden on health care resources. Few studies have investigated recent trends in hospital admissions and procedures for SDD in China. This study aimed to identify the trends, if any, in the number of surgical procedures undertaken for SDD in a large patient cohort. Materials and methods: This retrospective cohort analysis used data from inpatient medical records at Peking University Third Hospital between 2003 and 2016. Descriptive statistical analysis, regression models, and a Holt-Winters seasonal model were used to analyze trends. Results: Altogether, 38,676 surgery records from 37,897 SDD patients who had undergone surgical treatment were included in our study, among whom $49.60 \%, 47.81 \%$, and $2.59 \%$ were treated because of cervical, lumbar, and thoracic degenerative disease, respectively. There was an increasing trend for spinal surgery performance with an increasing mean age at surgery, from 50.65 years of age in 2003 to 55.29 years in 2016. We also revealed interesting seasonal variation in our study - that is, most of the spinal procedures were performed during the winter and spring months.

Conclusion: Our study showed a significantly increasing surgical workload for addressing SDD in China. Both the public and the health care system should be aware of this increase in chronic degenerative disease in the aging population.

Keywords: spinal degenerative disease, surgical treatment, trends, seasonal variation

\section{Introduction}

By 2016 , as much as $11.40 \%$ of the Chinese population was over 65 years of age, which comprises one of the most important public issues in China today. ${ }^{1}$ Accompanying this aging population is chronic degenerative disease, which might require spinal surgery. Its incidence is expected to increase along with the increasing aged population. ${ }^{2}$ Studies worldwide have reported similar trends attributable to the country's particular demographics and health care systems. In developed countries, such as England and the USA, dramatically increasing trends in surgery for degenerative lumbar spine disease have been identified. ${ }^{3,4}$

China can claim the largest aging population in the world and is now facing the increasing burden of spinal degenerative disease (SDD); yet, few published studies have investigated the longitudinal trends in surgical procedures for SDD in China. In addition, although not widely accepted, some studies suggested that the risk of developing a surgical site infection (SSI) associated with spine surgery is higher 
during summer., 5 To our best acknowledge, however, no study has focused on possible seasonality effect on spine surgery in the Chinese population.

Therefore, we aimed to examine trends in spine surgery, particularly those for SDD, over the past decade to clarify the demographics of the disease and its treatment patterns. We also explored a possible seasonal variation for this surgery.

\section{Materials and methods}

Participants

In northern China, the Department of Orthopedics at Peking University Third Hospital is the major center for spine surgery, which ranks among the top three centers nationally in the annual number of spinal operations performed. For the present study, the inpatient medical records from our Department of Orthopedics from January 1, 2003 to December 31, 2016 were evaluated. Only hospitalized patients with SDD who underwent spinal surgery were enrolled in this study. Non-Chinese patients and those under 19 years of age were excluded. SDD patients who had been hospitalized and undergone surgery more than once were included in the study, but using only the data from their first hospitalization.

The Ethics Committee of Peking University Third Hospital approved this study (IRB00006761-M2018082). The study was performed under the guidance of the Helsinki Declaration for protecting the confidentiality of patient data. Participant informed consent was waived because this research involved no more than minimal risk of exposure.

\section{Data source}

The age, sex, and diagnosis of the patients were retrieved from their medical records. SDD diagnosis was subdivided into cervical (CDD), thoracic (TDD), and lumbar (LDD) degenerative diseases. Doctors in the orthopedics department made the diagnosis, and it was coded by the staff of the Medical Records Department based on the ICD, Tenth Revision, Clinical Modification (ICD-10). The codes relevant to SDD in our study were as follows: M47.0, M47.1, M47.2, M47.8, M47.9, M48.0, M48.9, M50.0, M50.1, M50.2, M50.3, M51.0, M51.1, M51.2, M51.3. Inpatient medical records in this research were collected from the Peking University Third Hospital electronic medical system by searching ICD-10 diagnosis codes. Patients who had not undergone surgery were excluded.

\section{Statistical analysis}

Descriptive analysis was performed to reveal the composition of the SDD subtypes, between sex and different age groups.
The regression analysis focused on accessing the trends in mean age at surgery, sex, and average hospitalization day during the study period. Regression models (linear and inverse proportion regression) were used, with demographic characteristics as the dependent variables and the calendar year index as the independent variable.

We also used time series analysis to explore the possible seasonal fluctuation in the number of spinal operations by adopting the Holt-Winters (HW) seasonal additive model. We split the total time series into two subsets before the final calculation: the training set and the testing set. The analysis was performed as follows:

1. Data preparation: The SDD patients enrolled monthly who underwent a spinal operation from January 1, 2003 to December 31, 2015 were considered for the training set, whereas the remaining patients (from January 1, 2016 to December 31, 2016) formed the testing set. Both training and testing sets were converted to time series.

2. Model was trained (Forecast package is available for an exponential smoothing purpose).

3. Verification that the model was sufficient and could not be improved (Ljung-Box [LB] test was used to assess residual autocorrelation).

All analyses were performed in $\mathrm{R}$ for Windows 3.4.3. A value of $P<0.01$ was used to indicate statistical significance.

\section{Results}

Overall, we collected 38,676 surgical records from 37,897 patients diagnosed with SDD (ie, CDD, TDD, and LDD). Among them, we found 779 patients whose disease involved various spinal segments and therefore underwent multiple surgical operations during the study period. We focused on analyzing the 37,897 patients who underwent surgery for their SSD, distinguishing them according to the affected site (cervical, thoracic, and lumbar).

CDD and LDD comprised $49.60 \%$ and $47.81 \%$ of all the affected sites, respectively, and TDD accounted for only $2.59 \%$. The differential distribution of the SDD subgroups was assessed according to the patients' age and sex. The female/male distribution of LDD and TDD were similar (24.57\% vs $23.24 \%$ and $1.18 \%$ vs $1.41 \%$, respectively). In the CDD group, however, $17.74 \%$ were female and $31.86 \%$ were male. Most operations were performed in patients $41-65$ years of age: $32.74 \%$ for CDD, $31.37 \%$ for LDD, and $1.93 \%$ for TDD (Table 1). Further analysis of the distribution of SDD by age showed that it was similar at every age group: CDD (50.0\% at $19-40$ years of age, $50.0 \%$ 
Table I Surgical operations for spinal degenerative diseases, by patients' sex and age

\begin{tabular}{|c|c|c|c|c|}
\hline & Category & $\begin{array}{l}\text { CDD } \\
\text { n (\%) }\end{array}$ & $\begin{array}{l}\text { LDD } \\
\text { n (\%) }\end{array}$ & $\begin{array}{l}\text { TDD } \\
\text { n (\%) }\end{array}$ \\
\hline \multirow[t]{3}{*}{ Sex } & Female & $6,724(17.74)$ & $9,313(24.57)$ & $448(1.18)$ \\
\hline & Male & $12,073(3 \mid .86)$ & $8,806(23.24)$ & $533(1.4 I)$ \\
\hline & \multicolumn{4}{|c|}{ Chi-squared test, $P$-value $<0.01$} \\
\hline \multirow[t]{4}{*}{ Age category } & $19-40$ & $3,511(9.26)$ & $3,447(9.10)$ & $120(0.32)$ \\
\hline & $41-65$ & $12,407(32.74)$ & II,888 (31.37) & $733(1.93)$ \\
\hline & $66+$ & $2,879(7.60)$ & $2,784(7.35)$ & $128(0.34)$ \\
\hline & \multicolumn{4}{|c|}{ Chi-squared test, $P$-value $<0.01$} \\
\hline Total & SDD & I8,797 (49.60) & $18,119(47.81)$ & $981(2.59)$ \\
\hline
\end{tabular}

Abbreviations: CDD, cervical degenerative diseases; LDD, lumbar degenerative diseases; SDD, spinal degenerative disease; TDD, thoracic degenerative diseases.

at $41-65$ years, and $50.0 \%$ at $\geq 66$ years) and LDD ( $49 \%$ at $19-40$ years, $47 \%$ at $41-65$ years, and $48 \%$ at $\geq 66$ years). However, the percentage of TDD was slightly higher in those aged $41-65$ years than the other two age groups ( $2 \%$ at $19-40$ years, $3 \%$ at $41-65$ years, and $2 \%$ at $\geq 66$ years), as shown in Figure 1.

Further analysis revealed that the gap between the sexes regarding the spinal procedures narrowed over the 14 years of the study. In 2003 , only $37.59 \%$ of the operations were performed in women, which increased to $46.41 \%$ in 2016 , and the trend continued (linear regression: $\beta=0.007$, adjusted $R^{2}=0.80, P<0.01$; Figure 2$)$. In addition, the mean age at the time of surgery for all SDD patients increased from 50.65 years in 2003 to 55.29 years in 2016 , with an estimated annual increase of 0.27 years $\left(\beta=0.27\right.$, adjusted $R^{2}=0.82$, $P<0.01$; Figure 3).

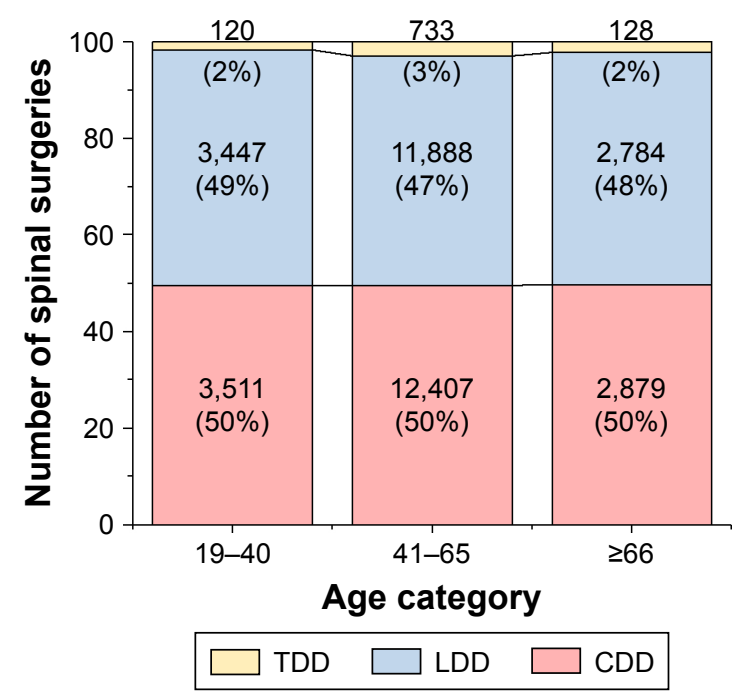

Figure I Number of spinal operations for SDD, by age group. Abbreviations: CDD, cervical degenerative disease; LDD, lumbar degenerative disease; SDD, spinal degenerative disease; TDD, thoracic degenerative disease.
In $2003,29.61 \%$ of the SDD surgery was carried out in patients aged $19-40$ years, but it decreased to $13.03 \%$ in 2016. Comparatively, the proportion of spinal surgery performed on patients aged 41-65 years showed a slight increase (from 59.91\% in 2003 to $66.92 \%$ in 2016), and a more conspicuous increase occurred in those aged $\geq 66$ (from $10.48 \%$ in 2003 to $20.05 \%$ in 2016 ).

The mean hospital stay decreased dramatically during the study period - from 18.76 days at the beginning of the study period to 7.79 days in 2016 , representing a fall of 0.78 day per study year (inverse proportional regression, $P<0.01$; Figure 4). During the study period, there was a significant increase in the overall number of operations for SDD - from 878 in 2003 to 3,277 in 2016, with an average annual increase of $12.32 \%$. The number of spinal surgeries showed temporal variation (HW time series analysis: $\mathrm{LB}$ test $P=0.18$; Figure 5). Taking a deeper look, the time series data showed that the increase in the number of surgical operations during the study period was not stationary, but exhibited an ascending

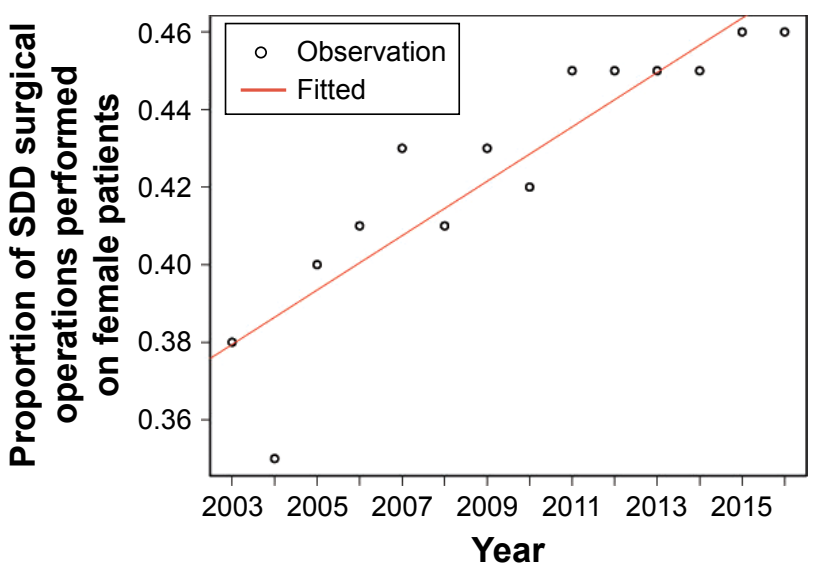

Figure 2 Proportion of SDD surgical operations performed on female patients, by the years of the study.

Abbreviation: SDD, spinal degenerative disease. 


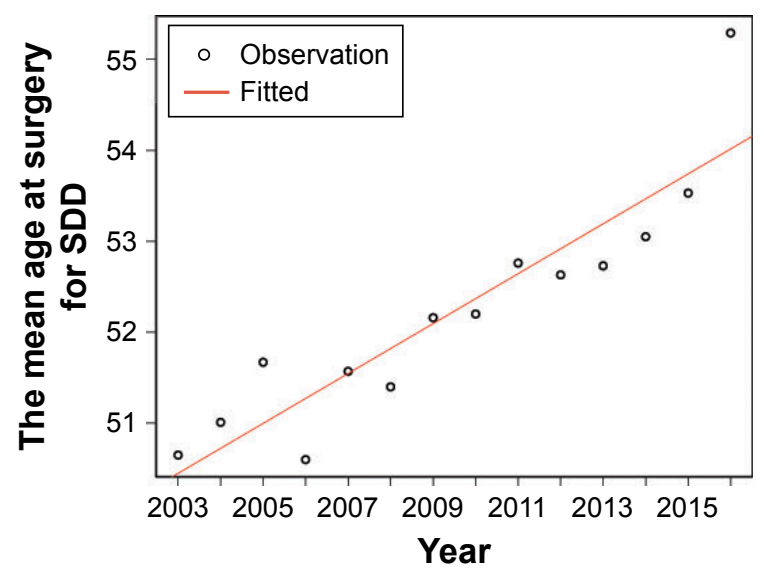

Figure 3 Mean age at surgery for SDD, by the year of the study. Abbreviation: SDD, spinal degenerative disease.

trend and seasonal behavior. The number of spinal operations always peaked during the winter and spring months (the highest numbers were during March, April, and November and the lowest number was in February).

\section{Discussion}

This study identified a significant increase in surgical procedures for SDD at our center from 2003 to 2016, with an increased mean age at surgery and decreased hospital stay for the surgical procedure. The gap between the sexes showed a narrowing trend, with the women accounting for an increasing proportion. We also identified seasonality as a factor in our study, suggesting aggregation of procedures be undertaken during the winter and spring months.

The Department of Orthopedics, Peking University Third Hospital is the largest spine surgery center in northern China, and thus likely represents the overall situation with surgical procedures for SDD in that region. Most of our findings are

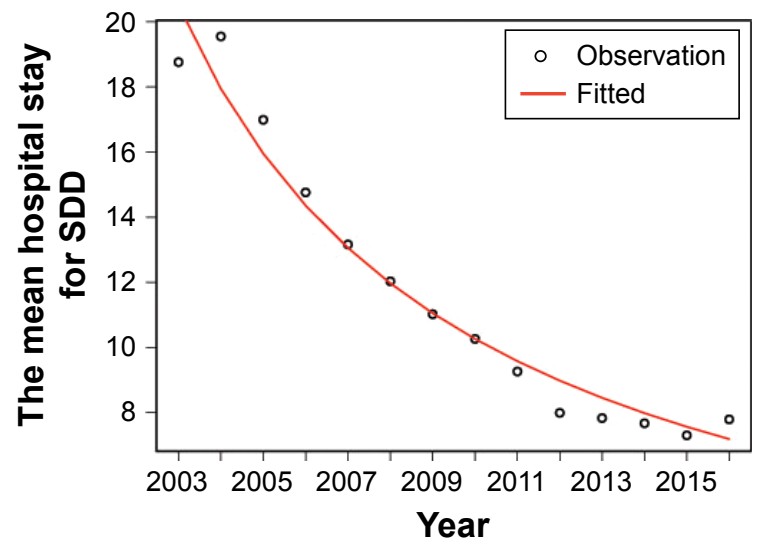

Figure 4 Mean hospital stay for postoperative SDD patients, by the years of the study.

Abbreviation: SDD, spinal degenerative disease.

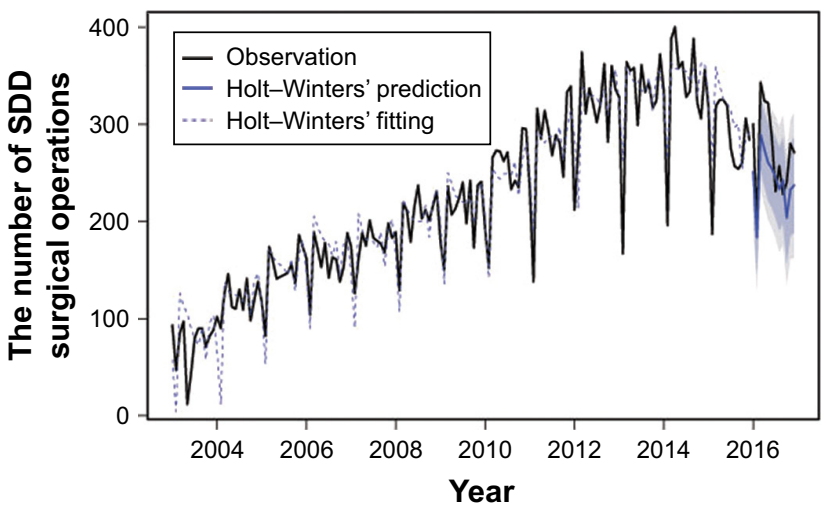

Figure 5 Number of observed, fitted, and predicted SDD surgical operations, by the year of the study.

Abbreviation: SDD, spinal degenerative disease.

consistent with studies performed in other countries. ${ }^{2,7,8}$ The trend for increasing spinal surgery has been identified in many countries and health care systems. ${ }^{2,9} \mathrm{We}$ also observed a shift in age to an older population for spinal surgery associated with an increase in the mean age at the time of surgery. The underlying reason for these changes could be aging of the population, which has been on a severely steep incline in China, as well as the overall improvement in the diagnosis and treatment of SDD. ${ }^{10}$ Other factors may also have played a part, such as more sedentary lifestyle, increased public awareness of health, and the rapidly rising rate of urbanization - the "epidemiological transition" - reflecting the evolution from parasitic and infectious diseases to chronic and degenerative diseases. SDD is one of the leading causes of visits to physicians and utilization of health care services in developed countries, ${ }^{2}$ conferring a huge burden on social and economic resources. The rapidly ascending trend of spinal surgery for SDD observed in our study may attract public attention to this chronic degenerative disease.

We identified a narrowing gap between the sexes for surgical treatment of SDD, although the percentage of men is still higher than that of women. Some studies have reported no differences in the incidence of SDD between the sexes, ${ }^{11}$ and a study in Korea showed that, among those $>60$ years of age, women are at greater risk for having SDD. ${ }^{12}$ Similar findings have been reported for England that hospitalization of female SDD patients increased more rapidly than that of male SDD patients. ${ }^{2}$ This trend may be attributed to the longer life expectancy of women, as the average life expectancy in China increased by 4.94 years from 2000 to 2015 overall, but increased by 6.10 years in the female cohort. ${ }^{1}$

We also identified an interesting seasonal variation in our study. The SDD surgical procedures were performed 
mostly during the winter and spring months, with lesser number of procedures done during summer. It is reasonable for us to deduct that the time of the surgery might have been the surgeon's and patient's choices. Other studies revealed that the risk for SSI is higher in summer than during the remainder of the year. ${ }^{13,14}$ In contrast, another study in Japan showed that SSIs following spinal surgery are significantly more in April. ${ }^{6}$ Other studies failed to obtain similar results, and some even had contrary findings. ${ }^{15}$ Hence, so far, there is no consistent view on whether a particular season is a risk factor for an SSI after spinal surgery. Our study showed seasonal variation, but it will be up to future studies to determine whether SSIs after spinal surgery follow similar fluctuation pattern. The answer may influence our clinical practice as to whether we need more perioperative measures to control the possible high risk of SSIs during a certain season.

Our study had some limitations. First, because of the one-center data set, possible bias may exist. However, considering that Peking University Third Hospital is one of the top spinal surgery centers in China and our results mirror trends identified in other studies, this study likely reflects the overall situation in China. Second, based on the ICD-10 diagnosis for defining SDD, this study lacks radiological evaluation for these SDD patients who underwent surgery. Nevertheless, there is evidence to prove that the radiological degenerative changes are not necessarily associated with symptom severity, uncontrollable pain, or neurological deficit, which are the clinical indications for spine surgery. ${ }^{16}$ Third, our study does not directly address the underlying reasons for the observed trends. The study revealed only the real-life situation from a large data set, although numerous factors have been suggested that could cause the trends we found, including the urbanization of China in recent years, an increasingly sedentary lifestyle, and a growing aging population, among others. The trends identified in this real-world study may require a factorial study to better understand the driving factors behind them.

\section{Conclusion}

This investigation identified an increasing trend of spinal surgery in China and addressed the issue of the increased mean age of the patients undergoing this surgery. Future studies could focus on the underlying reason(s) for, and associated outcome(s) of, the seasonal variation in spinal surgery. Meanwhile, awareness of the trends may attract public attention and strengthen the primary prevention of SDD in China.

\section{Acknowledgment}

The authors thank Dr Wei Zhu (Peking University Third Hospital) for helpful comments on the manuscript and Dr Wei Liu (Peking University People's Hospital) for editing assistance. This work was supported by the National Natural Science Foundation of China (Grant No 81601573), the National Key Research \& Development Plan of China (Grant No 2016YFC0901901), the Chinese Academy of Medical Sciences (Grant No 2017PT63010, 2018PT33024), and the National Clinical Key Specialty Construction Project.

\section{Disclosure}

The authors report no conflicts of interest in this work.

\section{References}

1. China National Bureau of Statistics. Available from: http://www.stats. gov.cn. Accessed October 19, 2018.

2. Tetreault L, Goldstein CL, Arnold P, Harrop J, Hilibrand A, Nouri A, Fehlings MG. Degenerative cervical myelopathy: a spectrum of related disorders affecting the aging spine. Neurosurgery. 2015;77:51-67.

3. Sivasubramaniam V, Patel HC, Ozdemir BA, Papadopoulos MC. Trends in hospital admissions and surgical procedures for degenerative lumbar spine disease in England: a 15-year time-series study. BMJ Open. 2015;5(12):e009011.

4. O'Lynnger TM, Zuckerman SL, Morone PJ, Dewan MC, VasquezCastellanos RA, Cheng JS. Trends for spine surgery for the elderly: implications for access to healthcare in North America. Neurosurgery. 2015;77(Suppl 4):S136-S141.

5. Durkin MJ, Dicks KV, Baker AW, et al. Postoperative infection in spine surgery: does the month matter? J Neurosurg Spine. 2015;23(1):128-134.

6. Ohya J, Chikuda H, Oichi T, et al. Seasonal variations in the risk of reoperation for surgical site infection following elective spinal fusion surgery: a retrospective study using the Japanese diagnosis procedure combination database. Spine. 2017;42(14):1068-1079.

7. Buser Z, Ortega B, D'Oro A, et al. Spine degenerative conditions and their treatments: national trends in the United States of America. Global Spine J. 2018;8(1):57-67.

8. Weinstein JN, Lurie JD, Olson PR, Bronner KK, Fisher ES. United States' trends and regional variations in lumbar spine surgery: 1992-2003. Spine. 2006;31(23):2707-2714.

9. Deyo RA, Mirza SK. Trends and variations in the use of spine surgery. Clin Orthop Relat Res. 2006;443:139-146.

10. Fehlings MG, Tetreault L, Nater A, et al. The aging of the global population: the changing epidemiology of disease and spinal disorders. Neurosurgery. 2015;77(Suppl 4):S1-S5.

11. Steelman T, Lewandowski L, Helgeson M, Wilson K, Olsen C, Gwinn D. Population-based risk factors for the development of degenerative disk disease. Clin Spine Surg. 2018;31(8):E409-E412.

12. Oh $\mathrm{CH}$, Yoon $\mathrm{SH}$. Whole spine disc degeneration survey according to the ages and sex using Pfirrmann disc degeneration grades. Korean $J$ Spine. 2017;14(4):148-154.

13. Durkin MJ, Dicks KV, Baker AW, et al. Seasonal variation of common surgical site infections: does season matter? Infect Control Hosp Epidemiol. 2015;36(9):1011-1016.

14. Gruskay J, Smith J, Kepler CK, et al. The seasonality of postoperative infection in spine surgery. $J$ Neurosurg Spine. 2013;18(1):57-62.

15. Banco SP, Vaccaro AR, Blam O, et al. Spine infections: variations in incidence during the academic year. Spine. 2002;27(9):962-965.

16. Corniola MV, Stienen MN, Joswig H, et al. Correlation of pain, functional impairment, and health-related quality of life with radiological grading scales of lumbar degenerative disc disease. Acta Neurochir (Wien). 2016;158(3):499-505. 


\section{Publish your work in this journal}

Clinical Interventions in Aging is an international, peer-reviewed journal focusing on evidence-based reports on the value or lack thereof of treatments intended to prevent or delay the onset of maladaptive correlates of aging in human beings. This journal is indexed on PubMed Central, MedLine,

CAS, Scopus and the Elsevier Bibliographic databases. The manuscript management system is completely online and includes a very quick and fair peer-review system, which is all easy to use. Visit http://www.dovepress. com/testimonials.php to read real quotes from published authors. 